\title{
Politique
}

Politique

\section{Transformation des modes étatiques de contrôle social}

\section{Jules Duchastel et Danielle Laberge}

Numéro 20, automne 1991

URI : https://id.erudit.org/iderudit/040699ar

DOI : https://doi.org/10.7202/040699ar

Aller au sommaire du numéro

Éditeur(s)

Société québécoise de science politique

ISSN

0711-608X (imprimé)

1918-6584 (numérique)

Découvrir la revue

Citer cet article

Duchastel, J. \& Laberge, D. (1991). Transformation des modes étatiques de contrôle social. Politique, (20), 65-92. https://doi.org/10.7202/040699ar

\section{Résumé de l'article}

Cet article s'intéresse au problème des transformations récentes des formes étatiques de contrôle social. Avant même de statuer sur la réalité ou la profondeur de ces changements, les auteurs s'interrogent sur la différence entre les projets et la mise en œuvre de transformations. Ils attirent l'attention sur la complexité de ce qu'il et convenu de nommer le système pénal et, conséquemment, de ses transformations possibles. Distinguant les divers niveaux de cohérence entre éléments du système, ils montrent comment logiques politiques et bureaucratiques manifestent avant tout des tendances contradictoires.

Après avoir distingué cinq modalités d'intervention étatique dans le domaine du contrôle social - production législative et réglementaire, financement, coordination des activités des agences, supervision des activités des agences, gestion directe - ils présentent les principales transformations des pratiques discursives entourant le contrôle social et abordent quelques exemples d'évolution de ces activités de contrôle, en particulier le bénévolat et la privatisation
Ce document est protégé par la loi sur le droit d'auteur. L'utilisation des services d’Érudit (y compris la reproduction) est assujettie à sa politique d'utilisation que vous pouvez consulter en ligne.

https://apropos.erudit.org/fr/usagers/politique-dutilisation/ 


\title{
TRANSFORMATION DES MODES ÉTATIQUES DE CONTRÔLE SOCIAL
}

\author{
Jules Duchastel et \\ Danielle Laberge \\ Université du Québec à Montréal
}

\begin{abstract}
Cet article s'intéresse au problème des transformations récentes des formes étatiques de contrôle social. Avant même de statuer sur la réalité ou la profondeur de ces changements, les auteurs s'interrogent sur la différence entre les projets et la mise en œuvre de transformations. Ils attirent l'attention sur la complexité de ce qu'il et convenu de nommer le système pénal et, conséquemment, de ses transformations possibles. Distinguant les divers niveaux de cohérence entre éléments du système, ils montrent comment logiques politiques et bureaucratiques manifestent avant tout des tendances contradictoires.

Après avoir distingué cinq modalités d'intervention étatique dans le domaine du contrôle social - production législative et réglementaire, financement, coordination des activités des agences, supervision des activités des agences, gestion directe -, ils présentent les principales transformations des pratiques discursives entourant le controble social et abordent quelques exemples d'évolution de ces activités de contrôle, en particulier le bénévolat et la privatisation.
\end{abstract}

\section{Introduction}

La sociologie du contrôle social s'inscrit dans une tradition qui remonte à la fin du $X \mid X^{\bullet}$ siècle, plus précisément à la publication d'une série d'articles d'Edward Ross dans l'American Journal of Sociology, revue qui venait de paraitre'. II serait trop long de retracer ici les nombreuses transformations qu'a connues le concept de contrôle social $^{2}$. Disons simple-

1. Ces articles seront repris par Ross en 1901 dans un ouvrage trés connu : Social Control and the Foundation of Sociology.

2. Voir à ce sujet Ph. Robert, 1981. 
ment qu'il est souvent pris dans deux sens opposés. Ou bien il est assimilé à celui de reproduction sociale, ce qui lui fait perdre toute spécificité. Ou bien il renvoie aux divers modes de répression mis en œuvre exclusivement par l'État et il ne permet plus de saisir des situations de contrôle moins strictement répressives. Nous avons choisi une voie intermédiaire qui nous permet de définir le contrôle social a partir des situations ou des groupes auxquels il s'applique plus directement. Contrairement à la reproduction sociale élargie qui concerne l'ensemble des individus de la société, le contrôle social s'exerce, avant tout, sur des sous-ensembles (groupes ou individus) qu'il marginalise. Cette optique englobe differrentes formes de gestion ou d'intervention sociales qui ne se réfèrent pas nécessairement à des illégalités, mais qui renvoient cependant à la marginalisation de situations ou de comportements jugés déviants ${ }^{3}$.

Depuis une quinzaine d'années déjà, on constate dans la littérature - particulièrement la littérature de langue anglaise - un intérêt marqué pour les transformations que l'État apporte au système de contröle social. II en est de même dans plusieurs disciplines, de la criminologie au travail social, en passant par la sociologie du droit ou du contrôle social. Ces travaux reflètent le désenchantement qui a suivi l'échec relatif des projets de réforme des dernières années et une résignation devant les incitations à la rationalisation financière. En dehors des modèles interprétatifs particuliers, l'existence de transformations majeures dans les formes de contrôle social est considérée comme acquise et non problématique. De nombreux analystes posent à priori que les modes de

3. Malgré les critiques adressées aux usages du concept de contröle social, Cohen, 1983 le circonscrit à l'intérieur des paramètres suivants: «l will continue to use "social control" to refer to something narrower and more specific than the standard sociological and anthropological concept, yet something wider and more general than the formal legal apparatus for the control of crime. This in-between territory belongs to all organised responses to crime, delinquency and allied forms of deviance - whether sponsored directly by the state or by institutions such as social work and psychiatry, and whether designated as treatment, prevention, punishment or whatever* (p. 102). 
contrôle social ont subi des modifications et que ces modifications, loin d'être secondaires ou marginales, réorientent de façon importante les rapports effectifs entre la société civile et l'État, tel qu'il apparaît à travers différentes modalités spécifiques de contrôle social.

Ces analyses, comme celles qui touchent les autres domaines de l'intervention étatique, nous semblent poser des problèmes méthodologiques, sinon épistémologiques. Ces problèmes concernent l'articulation des divers ordres d'intelligibilité de la réalité sociale et les inférences implicites de l'un a l'autre. En voici deux exemples reliés à la représentation des transformations étatiques. Nous distinguerons les représentations conceptuelles ou theoriques qui servent de cadre d'analyse et les représentations sociales - discours étatiques ou idéologies - qui sont l'objet de cette analyse. Dans le premier cas, on se heurte a la rigidité de certaines catégories d'analyse prises comme des universaux plutôt que comme des constructions marquées par un contexte historique $^{4}$. II faut remettre en question l'usage des concepts qui nous servent de point d'appui, car ils sont soumis a l'évolution de la réalité qu'ils représentent. Par exemple, la signification des concepts d'étatisation, de privatisation ou de centralisation, bien que comportant un noyau sémantique relativement permanent, peut varier grandement en regard de l'évolution historique des réalités qu'ils recouvrent. Dans le cas des représentations sociales, il arrive trop souvent que l'on prenne le discours pour la réalité. Ainsi, sur la base de discours publics qui ont pour thèmes les nécessaires transformations des formes d'intervention étatique, on infère que ces transformations sont déjà appliquées dans les institutions, l'organisation ou les pratiques.

L'analyse des transformations du contrôle social nous semble devoir introduire une distinction entre l'énoncé des

4. Les travaux de Polanyi, tels que rapportés par Jean-Jacques Gislain, dans * L'État et le marché: réflexions sur leur articulation institutionnelle*, Interventions economiques, $n^{\circ} 17,1987$, suggèrent de renoncer à ce type d'approche, en dénaturalisant certains concepts (cornme celui de marché) et en rétablissant leur caractère historique. 
projets, sous forme d'intentions administratives ou de politiques spécifiques, et la mise en œuvre de ceux-ci. On pourrait parler d'un monde d'intentionnalités représentant le réel sur la seule base des discours, qui se construit en interaction avec un monde de pratiques spécifiques.

Un second ordre de problemes concerne la variation des types de transformation suivant les secteurs ou les formes de contrôle social. Peut-on dégager de larges tendances, des tendances importantes qui dépassent les variations sectorielles ou ponctuelles? La réponse nous semble résider dans le découpage de l'analyse, qui nous évitera d'en être réduits a des généralisations abusives basées sur des observations partielles. Sans un découpage de l'analyse, l'on arrive aux affirmations les plus contradictoires à propos des mêmes réalités, par exemple «y a moins d'État, mais plus de contrôlen.

Dans le présent article, nous ferons d'abord quelques clarifications qui nous apparaissent comme des préalables a une analyse des transformations du système pénal. Bien que notre démarche se situe dans le cadre plus large du contrôle social, conçu comme la gestion sociale de situations ou de groupes marginalisés, nous nous en tiendrons au domaine plus restreint de la pénalité. De façon précise, nous examinerons quels principes de cohérence sont ou ne sont pas dans l'articulation des différentes agences composant le système pénal. De plus, nous spécifierons les modalités particulières et distinctes d'intervention de l'État dans le domaine pénal. Enfin, d'un point de vue prospectif, nous tenterons d'identifier les caractéristiques des transformations du contrôle social étatique en distinguant les transformations discursives et les transformations matérielles.

\section{Clarifications}

Des critiques récentes ont souligné les difficultés que présentent les analyses globalisantes des dernières années (Bottoms, 1983; Cohen, 1987; Lascoumes, 1989a; Matthews, 1987; Young, 1983). Au lieu de reprendre les divers aspects de ces critiques, nous tenterons, dans la 
perspective qu'elles développent, de mettre en évidence certaines questions qui nous semblent essentielles pour notre analyse.

\section{Système pénal et principes de cohérence}

Nous retiendrons d'abord le problème que posent les expressions «système pénal», kappareil pénal» et «système de justice" ("rcriminal justice system»). En raison du principe unitaire et exclusif qu'elles évoquent, ces désignations prêtent à confusion. La question est de savoir s'il existe vraiment un système pénal. Un ensemble d'agences (police, tribunaux, instances correctionnelles, etc.) se partagent la gestion de la pénalité. Par commodité, on les désigne comme des constituants d'un système pénal. Bien qu'il existe une cohérence certaine dans les activités de nature pénale qui relèvent de ces différentes instances, cela n'implique pas pour autant qu'elles cumulent tous les ordres de cohérence qui caractérisent un système. Par conséquent, si l'articulation entre les différentes agences du système pénal est marquée par une cohérence d'ordres juridique et processuel, il ne faut pas en conclure qu'il y a cohérence sur les plans bureaucratique et politique.

Le premier ordre, celui de la cohérence juridique, représente en quelque sorte une "métacohérence". Cette cohérence découle du fait que toutes les activités pénales relèvent de l'ordre juridique et $y$ trouvent leur justification. Le droit constitue ici l'ultima ratio de chacune des agences et, par extension, du fait qu'on les envisage comme un système. Chacune des agences est fondée directement par et dans le droit, produit de la nécessité pour l'État de perpétuer l'ordre et de réprimer le crime. A ce titre, les agences peuvent être considérées comme les parties essentielles d'un tout qui leur donne leur cohérence.

Le second ordre de cohérence est de nature processuelle. II se réfère à la prise en charge des individus sur le plan pénal. La cohérence ici se situe dans la logique de l'itinéraire que doit suivre le citoyen qui est appréhendé. Le parcours à travers les agences se déroule selon un ordre prévu et est régi par des règles procédurales qui en déterminent la mécanique générale. 
C'est ainsi que la sentence suit le procès au lieu de le précéder. Mais cela n'implique nullement que tous les individus sont traités de la même manière au cours de ce processus partiellement réglés. Ce n'est donc pas de la nature ou des déterminants de la prise en charge dont il est question, mais plutôt de leur ordre, de leur organisation séquentielle, qui suppose une cohérence minimale entre les différentes agences. C'est donc à l'intérieur de ces deux ordres, juridique et processuel, qu'il est possible de concevoir le domaine pénal comme un système.

La cohérence interne, prémisse de cette reconstruction, est trop souvent étendue à des niveaux où elle n'existe pas. C'est là que se situe le problème. En effet, on suppose qu'il y a une coherence (des principes) politique dont la logique serait partagée par l'ensemble des instances concernées : tribunaux, police, instances de gestion des peines. On aurait affaire a un fonctionnement exclusivement "top-down" (Matthews, 1987), selon lequel les changements seraient la résultante de décisions prises aux niveaux supérieurs. Cette conception extrêmement centralisatrice et réactive du fonctionnement de l'appareil pénal ne correspond pas du tout à son fonctionnement réel (Lascoumes, 1989 a). Par exemple, autant au Québec qu'au Canada, des énoncés de politique définissent de nouveaux principes d'orientation en ce qui a trait à la réduction de l'utilisation du système pénal, a l'accroissement de la sélectivité des individus visés et a la diminution des peines. Malgré le caractère explicite et concordant de ces intentions politiques, aucune observation empirique ne permet de confirmer une réduction marquée, à I'un ou l'autre titre, de l'utilisation du système pénal durant cette période.

5. Certaines trajectoires spécifiques susceptibles d'être affectées par différentes caractéristiques des justiciables - le sexe, la classe sociale ou $l$ 'origine ethnique, pour ne nommer que les plus évidentes - ou contextuelles. Cette cohérence processuelle ne peut être assimilée en aucune façon a un traitement égalitaire des individus qui s'y trouvent. Voir entre autres Pires et Landreville, 1985, qui identifient au moins cinq facettes de l'inégalité juridico-pénale (p.108-110). 
Quant à l'hypothèse d'une cohérence de nature bureaucratique, elle nous semble également inappropriée. Puisque la prise en charge pénale des individus correspond à des parcours relativernent prévisibles à l'intérieur de chacune des instances et d'une instance à l'autre, les agences qui composent le système pénal devraient avoir une certaine cohérence bureaucratique commune. La logique bureaucratique se superposerait, en quelque sorte, a la cohérence processuelle. II n'en est pourtant pas ainsi. En voici les raisons.

D'une part, chaque agence s'inscrit avant tout dans une logique d'intérêts, d'obligations et de contraintes qui lui est propre. Par ailleurs, si la dimension pénale de leurs activités nous incite à voir les agences dans leur ensemble comme un système, cette dimension ne rend pas compte de toutes leurs activités. Nous pensons ici principalement aux modalités complexes de régulation relevant de la police et des tribunaux, lesquelles dépassent la stricte gestion de la pénalité. Par exemple, bien que dans nos perceptions le rôle de la police soit de lutter contre la criminalité, il est bien plus de maintenir l'ordre - circulation routière, paix sociale, secours aux personnes dans le besoin, etc. De la même façon, la fonction pénale n'est qu'une des attributions des tribunaux, et la division des juridictions témoigne de la diversité de ses sphères d'intervention. L'activité judiciaire ne peut donc être réduite à la seule activité pénale.

$D^{\prime}$ autre part, le parcours des différentes étapes de la prise en charge pénale montre les contradictions profondes qui existent entre les diverses agences et ne correspond pas nécessairement aux règles énoncées. Par exemple, les tribunaux, en vertu de leur autonomie relative, émettent une somme de sentences qui dépasse de beaucoup les capacités d'accueil des centres de détention, d'où la surpopulation des institutions carcérales, qui nécessite le recours à différentes stratégies ponctuelles. Entre autres, il faut trouver des moyens de remettre en liberté des incarcérés avant la fin de leur sentence. La surpopulation des institutions carcérales ne se manifeste pas seulement au Québec et au Canada, mais elle semble affecter presque tous les pays occidentaux la 
I'exception peut-être des pays dont les politiques pénales sont plus douces, tels que la Hollande et les pays scandinaves) ${ }^{\circ}$.

Modalités d'intervention étatique

La seconde source de confusion qui peut gêner l'analyse des transformations actuelles du système pénal provient de ce que l'intervention étatique est pensée globalement (Brodeur, 1988; Laberge, 1988 a). Puisque les formes particulières d'articulation entre l'État et l'appareil pénal sont univoques et non complexes, il devient difficile d'en identifier les transformations. En analysant les transformations étatiques, on s'en tiendra alors à des catégories binaires : présence/absence, privé/public, volontaire/coercitif, etc. Les représentations habituelles du développement du système pénal ont associé, de manière trop exclusive, le développement de l'État moderne et celui du système pénal. En conséquence, elles ont contribué à masquer la complexité de la construction progressive de cette imbrication entre l'État - dans les sociétés capitalistes avancées - et les formes les plus dures du contrôle social exercées par les agences pénales.

Dans le domaine qui nous intéresse, l'État contemporain intervient selon des modalités distinctes et spécifiques. II nous semble d'ailleurs que des clarifications a ce sujet devraient permettre de résoudre un certain nombre de contradictions apparentes dans les résultats des recherches effectuées au cours des dernières années. Certaines de ces recherches partent de la prémisse d'un accroissement, d'une diversification et d'une diffusion du contrôle social, alors que d'autres supposent le mouvement inverse, c'est-d-dire le retrait de l'État, par l'abandon de quelques domaines d'intervention.

6. Voir entre autres la section xdébat» de la revue Déviance et société consacrée à ce phénomène, 1988, vol. XII, n 3 . 
Nous ramènerons à cinq modalités distinctes les interventions de l'État dans le domaine de la gestion sociale' : la production législative et réglementaire, le financement, la coordination des activités des agences, leur supervision et la gestion directe.

La production législative et réglementaire correspond aux activités servant à déterminer des obligations ou des prohibitions qui concernent les activités des individus, des groupes ou des organisations. Les énoncés sont assortis de sanctions qu'entraîne leur violation. La réglementation crée aussi les conditions de sa propre mise en œuvre, c'est-à-dire qu'on y prévoit les modalités de son application par la création, la définition, la réorganisation et, parfois même, l'abolition d'agences (au sens d'instances) administratives.

Le financement est probablement l'activité la plus facile à saisir. Nous regroupons sous cette rubrique toute forme de déboursés assumés par l'État, et permettant l'instauration ou l'exercice d'activités. Au financement des services directement sous juridiction gouvernementale, on peut ajouter le transfert direct par contrats de service ou subventions, les avantages fiscaux pour les entrepreneurs, et les programmes particuliers de subsides aux entreprises".

La coordination des activités dévolues aux agences est une tâche étatique essentielle qui assure la cohérence processuelle dont il a été question. Si, habituellement, l'autorité exclusive de chaque agence ou instance administrative est fixée de façon réglementaire, sa délimitation reste insuffisante pour que les citoyens soient dirigés efficacement dans le réseau des services. L'État doit donc développer des mécanismes de coordination particuliers.

7. Le concept de gestion sociale doit être distingué de celui de contrôle social de deux manières. D'abord, il renvoie a une réalité plus large (la reproduction sociale) et ne concerne pas exclusivement les situations ou groupes marginaux. Ensuite, il désigne plus spécifiquement les dispositifs de mise en œuvre de la reproduction sociale et, plus précisément ici, du contrôle social.

8. Voir Warren, 1981, sur les formes diverses de financement pouvant etre mises en cuvre apress la privatisation. 
La supervision des activités des agences, en tant qu'intervention spécifique de l'État, n'est pas toujours évidente parce que souvent les fonctions de gestion directe $\left(5^{\circ}\right.$ modalité) et de supervision sont concomitantes. Depuis la dissociation récente entre la gestion des clientèles et la mise en œuvre générale des politiques, il est possible de reconnaître plus clairement l'importance de la supervision. Par ce biais, l'on s'assure que la réglementation ou certains aspects de celle-ci sont respectés de façon minimale.

L'intervention étatique se manifeste enfin sous forme de gestion directe, $c^{\prime}$ est-a-dire de prise en charge effective des individus par les agences étatiques. Au sens strict, c'est l'État-entrepreneur, l'État-intervenant dont il est ici question. Dans le domaine pénal, l'État a mobilisé la quasi-totalité des activités d'intervention directe et de prise en charge. La fusion n'est pas aussi marquée dans d'autres secteurs de la gestion sociale.

Pour chacune des agences ou, de façon plus large, pour chacun des types d'activités composant le système pénal, l'une ou l'autre des dimensions ci-dessus sera plus ou moins pertinente. En effet, seule la production légale et réglementaire appartient entièrement à l'État dans le domaine qui nous intéresse. Celui-ci intervient toujours sur la base d'une réglementation prise au sens large. En revanche, les autres modes d'intervention possibles, de forme variable, sont toujours présents, puisqu'il est difficile d'imaginer qu'aucune modalité ne mette en œuvre l'acte législatif. Comme l'a clairement montré Gurvitch (1940), la règle juridique produit des effets symboliques en dehors de sa mise en œuvre effective. Une loi peut avoir des effets sans qu'aucune modalité ne soit prévue pour son application, mais son impact est alors restreint. Il est difficile de concevoir qu'elle ait un pouvoir de structuration ou de normalisation si elle ne donne lieu à une application même partielle et incomplète ${ }^{9}$.

9. II ne s'agit pas de prétendre ici à une cohérence parfaite ou même importante entre le droit «fictif» et le droit en ceuvre. Mais il faut au minimum qu'existe une certitude quant à la potentialité du second pour qu'agisse le premier. 
L'analyse des mouvements que subit le fonctionnement des différentes agences chargées du contrôle social, et plus spécifiquement le fonctionnement du sytème pénal, se heurte toujours à un problème épineux. Comme l'a fait remarquer si justement Matthews (1987), l'État se trouvant au cœur même des pratiques de contrôle, il est facile d'interpréter les transformations importantes qui le concernent comme source de toute transformation dans les modes de contrôle social. Un tel glissement permet de faire l'économie d'analyses spécifiques et de transposer automatiquement toute transformation globale de l'État au niveau d'activités partielles comme les pratiques de contrôle social. De façon bien élémentaire, il faut essayer de distinguer deux opérations : I'identification des transformations et les explications concernant la source de ces transformations.

Dans les pages qui suivent, nous essaierons d'identifier parmi les modifications apportées aux pratiques celles qui nous apparaissent comme des signes de transformation véritable et non comme de simples ajustements fonctionnels. Nous distinguerons les pratiques d'ordre discursif lénoncés de politiques, thématiques de débats, propositions d'orientation) et les pratiques effectives. II est essentiel de faire la différence entre les transformations discursives et les transformations concrètes quand le système n'a pas de cohérence politique, autrement dit quand il n'y a pas de concordance directe ou univoque entre des énoncés d'intention et leur mise en œuvre.

\section{Transformations des pratiques discursives : les thèmes importants}

Les thèmes que nous présentons renvoient aux principaux enjeux mis de l'avant depuis une quinzaine d'années dans le domaine de l'analyse du contrôle social par l'État et, plus spécifiquement, des procédures pénales. Cette thématique parcourt l'ensemble des sources discursives et caractérise aussi bien les énoncés d'intention politique et les productions gouvernementales que les analyses provenant des milieux scientifiques ou des milieux d'intervention. Par ailleurs, ces 
thèmes ne s'articulent pas spécifiquement ou exclusivement a un projet social, a une position politique ou à un modele interprétatif particulier, mais couvrent, sous une forme ou une autre, l'éventail des modèles d'interprétation ou des options sociopolitiques. Nous avons retenu trois axes thématiques distincts : communauté et responsabilisation; privatisation, économie et efficacité; technologie, surveillance et sécurité.

\section{Communauté et responsabilisation}

Depuis une quinzaine d'années déjà, les idées de communauté et de responsabilité individuelle ou collective émergent dans les projets politiques et dans les analyses scientifiques. L'aspect intéressant est que ces idées sont une remise en question de l'identité des responsables de l'intervention. En effet, le $X X^{*}$ siècle a été marqué par l'accroissement constant de l'intervention directe de l'État dans les différents secteurs du contrôle social. Par l'intermédiaire des agences étatiques, l'État s'est graduellement érigé en titulaire de l'intervention dans une multitude de situations sociales ou individuelles. C'est pourquoi l'échec des projets réformistes ou novateurs au cours des années 70 a été attribué à une trop grande emprise de l'État. Cela explique l'apparition, dans les nouveaux projets, d'un principe de substitution ou d'atténuation de la place qu'il occupe.

L'importance de plus en plus grande que revêtent dans les discours politiques et scientifiques les notions de communauté, de responsabilité individuelle ou de responsabilité familiale constitue un pôle d'opposition aux formes étatiques d'intervention. Elles se présentent comme un modèle possible et informel - c'est-à-dire dont les modalités ne sont pas définies - de substitution a l'État. Ce modele, basé sur des représentations sociales extrêmement puissantes, suscite des émotions à la fois positives et floues (Cohen, 1987; Laberge, $1988 a, b)$.

Par ailleurs, il est certain que les notions de responsabilité individuelle ou familiale et de communauté peuvent s'inscrire dans des projets politiques très différents. Leur prédominance dans les débats des dernières années vient probablement du fait qu'elles sont polysémiques et que chacun peut y projeter 
ce qu'il veut. La mise de l'avant de l'idée de communauté s'appuie en effet sur des plans d'analyse très différents, dont les plus évidents se retrouvent dans les couples d'oppositions suivants : local/centralisé; personnalisé/bureaucratique; informel/formel; souple/rigide; mais, aussi, modération/abus de service; autonomie/dépendance. D'autre part, la présentation des thématiques de communauté et de responsabilité a rarement dépassé le stade du slogan ou de l'idée intéressante. Ainsi, la notion même de communauté n'est pas construite de manière élaborée ou explicite dans les projets proposés ${ }^{10}$. Ce qui a permis qu'elle soit utilisée de diverses façons et lui a évité des remises en question.

\section{Privatisation, économie, efficacité}

Le second axe thematique, vu comme une source de transformation sociale, concerne principalement le financement des interventions et leur caractère plus ou moins performant. II renvoie aux notions de privatisation, d'économie, de saine gestion et d'efficacité. L'émergence de ces préoccupations coïncide avec la crise fiscale que connaissent la plupart des gouvernements des pays occidentaux. Remontée du néo-libéralisme, rationalisation budgétaire des dépenses publiques, déréglementation, voilà autant de tendances qui ont touché aussi les activités de contrôle social. II ne s'agit pas de déterminer si cette crise est une source de transformations ou si celles-ci ne constituent qu'une des manifestations de la crise. Disons que dans le contexte de l'époque, encore actuel à beaucoup d'égards, les discours sur l'efficacité ou les vertus de l'entreprise privée ont marqué l'examen de toutes les questions sociales.

Cette thématique, tout autant que la première, a suscité beaucoup de débats sans pour autant évoquer chez tous des

10. Le meilleur exemple de cette absence de construction concerne le domaine de la désinstitutionnalisation psychiatrique qui est en œuvre depuis de très nombreuses années en Amérique du Nord. "I est impossible de trouver des projets sérieux de définition de ce qu'est la communauté qui apparaît, par défaut, comme étant tout lieu autre que l'hôpital psychiatrique (Laberge, 1988b; Lefebvre, 1985, 1987). 
images toujours positives". Nous croyons que c'est autour de ces questions que le pénal se distingue de façon claire des autres formes de contrôle social étatique. Si les personnes qui sont l'objet d'une intervention pénale sont représentées couramment comme les moins méritoires - d'autant moins méritoires qu'on les compare à d'autres groupes nécessitant un soutien public : les personnes agées, les malades, les personnes handicapées, etc. - , ce sont paradoxalement les agences pénales qui sont les moins menacées par les coupures budgétaires. II y a rupture totale entre l'idéologie du mérite, du besoin et les investissements effectifs. La priorité est donnée aux prétendues nécessités de l'ordre social.

Comme le faisait remarquer Brodeur (1988), le domaine du privé ne peut se réduire à une définition simple où se confondent sphère privée et entreprise privée. Ainsi, la privatisation $^{12}$ suppose-t-elle, dans son sens premier, le transfert de responsabilités de certaines agences étatiques à des instances non étatiques dont le statut peut être par ailleurs diversifié. Quelques cas viennent immédiatement à l'esprit : des entreprises poursuivant des buts lucratifs, des organismes sans but lucratif, des individus rémunérés ou non rémunérés.

A cette première source de diversification, c'est-à-dire l'identité des nouveaux responsables de l'intervention, s'en ajoute une seconde qui concerne la nature du passage dont

11. On peut, par spéculation, voir les projets de privatisation comme servant surtout des fins idéologiques qui s'adressent soit aux bénéficiaires, soit au personnel de ces agences. La privatisation se présentant souvent sous forme de coupures de postes ou de services, la menace de son instauration éventuelle sert ainsi $d$ 'incitatif puissant à la pratique de comportements d'autorestriction. On demandera moins de services, on en demandera moins souvent, ou alors on tentera d'être plus efficaces ou plus performants.

12. La privatisation renvoie implicitement à la notion d'intervention et de responsabilité pour l'intervention. Bien que nous n'en débattions pas explicitement ici, l'intervention dans le domaine pénal s'arrime nécessairement au cadre juridique qui la fonde. Là aussi des mouvements importants se font sentir, qui ne sont pas sans rapport avec certaines formes de privatisation. Pour une clarification de ces questions, voir $M$. Van de Kerchove, $*$ Les différentes formes de baisse de la pression juridique et leurs principaux enjeux». 
nous avons parlé. Encore une fois, on a trop souvent tendance à réduire l'idée de privatisation à sa manifestation la plus patente, qui est le transfert pur et simple des responsabilités du secteur public au secteur privé. Pourtant, divers cas s'inscrivent sous cette rubrique. La mise en place, par exemple, de nouvelles activités qui sont confiées à des instances non étatiques et l'expansion des responsabilités confiées aux instances privées sont d'autres changements qui très souvent décrivent beaucoup plus adéquatement les mouvements de transformation.

Pourtant, tous ces débats, la volonté de certains groupes de tenter des expériences, les incitations à une rationalisation des dépenses publiques dans ce domaine («la justice coûte cher»), ne peuvent faire l'objet d'un consensus prima facie, parce qu'ils sont indissociables de conceptions beaucoup plus fondamentales liées à l'exercice du droit et à l'obligation de punir. La renonciation à certains privilèges qu'implique l'idée de contrat social se fait au profit d'un État garant d'égalité et de modération. Ainsi, l'exercice de la capacité de punir et de réprimer ne peut être soumis aux lois du marché sans altérer fondamentalement la nature des liens symboliques qui créent à la fois l'État et la société civile.

Technologie, surveillance, sécurité

Le troisième thème touche à la sécurité, au contrôle et à l'impact des techniques. Ces aspects intimement liés aux précédents reflètent clairement les tensions traditionnelles entre les nécessités de l'ordre et le respect des droits individuels. Cette opposition s'est complexifiée avec le développement et l'utilisation accrue des nouvelles techniques de surveillance. L'accroissement de la sécurité ${ }^{13}$ semble se faire sans douleur et ne pas entraîner un usage plus grand de mesures explicitement répressives.

13. Dans une large mesure, on peut se demander s'il ne s'agit pas avant tout du sentiment de sécurité, qui serait en croissance, plutót que de la sécurité réelle, cette dernière pouvant d'ailleurs être définie de multiples taçons potentiellement contradictoires. 
Cette situation a tout de même de nombreuses conséquences. En premier lieu, elle permet à la transgression des droits individuels de se faire sous des modes indirects, non violents et même invisibles. La question qui devrait alors se poser de façon aiguë est de savoir si, par le biais de ces nouvelles stratégies de surveillance et de contrôle, l'on substitue vraiment des moyens moins sévères à d'autres plus sévères pour arriver aux mêmes fins. En second lieu, cette question fait surgir des problèmes moraux très difficiles a résoudre : Quel prix devrait-on payer pour éviter à certains individus des mesures extrêmement répressives? Qui devrait faire ce choix? Si l'usage de nouvelles méthodes de surveillance et la multiplication des stratégies dites préventives semblent poser des problèmes éthiques à la société, plusieurs en vantent l'efficacité. En effet, l'usage des techniques de surveillance $s$ 'inscrit directement dans la logique d'efficacité et d'économie dont plusieurs se font les défenseurs acharnés. Du point de vue de nombreux gestionnaires, c'est par ce biais que l'on pourra faire, à moindres coûts, des opérations aussi et même plus efficaces.

\section{Modèles idéals de transformation}

Les trois axes thématiques abordés ci-dessus signalent des transformations importantes des enjeux, projets et représentations dans le domaine pénal. Si, comme nous le disions précédemment, aucun projet social, aucune position politique, aucun modèle interprétatif ne peuvent prétendre monopoliser ces thèmes ou les articuler de manière très explicite, il est possible d'identifier des modèles idéals qui trouvent certains échos dans la société. La conjonction de ces thèmes donne naissance, en effet, à des modèles généraux de transformation, dont on ne sait souvent plus très bien quelle portion est prospective et quelle portion est déjà en œuvre.

\section{Modèle conservateur-libertaire}

Ce modèle met l'accent sur la responsabilité individuelle ou familiale et sur la nécessité pour l'État de se retirer de la 
quasi-totalité des activités de gestion directe, du moins dans le domaine correctionnel : la gestion des prisons par des entreprises privées et l'autofinancement des prisonniers; l'usage de nouvelles techniques pour surveiller certaines catégories de condamnés ou les personnes sous libération conditionnelle - a la fois sur la base de leur efficacité totale et de leurs couts minimes (Landreville, 1987) - ; et l'utilisation accrue de forces de sécurité privées. L'État est conçu avant tout comme le garant de l'ordre et de la sécurité. Les citoyens quant à eux doivent assumer leurs responsabilités.

\section{Modèle réformiste-écologiste}

Ce second modele suppose une transformation des formes d'intervention les plus dures, un usage plus discret de l'appareil pénal, le recours à des mesures communautaires, une décentralisation des lieux d'exercice du pouvoir, une limitation de l'intervention aux cas les plus graves et donc un accroissement de l'efficacité des pratiques de contrôle. Ici, la nécessité de l'intervention étatique n'est pas véritablement remise en cause; ce sont plutôt les formes ou les modalités de l'intervention qui sont critiquées. On fait une place importante a l'idée de partenariat - pas toujours sous ce vocable - au moyen d'une coordination plus étroite des activités entre des regroupements communautaires.

Modèle radical-critique

Un troisième modèle interprète les récents développements comme des signes d'extension du contrôle social, entre autres par l'ajout de nouvelles mesures souvent plus douces ("widening of the net") et par l'usage accru de techniques de surveillance. Le modèle radical-critique présente une société dans laquelle le nombre des individus qui font l'objet de contrôle immédiat va en s'accroissant gråce à des mesures toujours plus nombreuses et plus subtiles de surveillance ou de prise en charge. Selon cette hypothèse, la mise en place 
de mesures alternatives ${ }^{14} n^{\prime} a$ pas un caractère substitutif mais plutôt additif. II $n^{\prime} y$ aurait donc pas de façon de contrer l'envahissement progressif de l'État.

L'examen des transformations discursives permet donc de voir les enjeux, les ruptures, les contradictions que contiennent plus ou moins explicitement les projets sociaux. D'autre part, ces débats, et les modèles auxquels ils renvoient, "déteignent" nécessairement sur les pratiques effectives - et ce, en dehors de toute transformation matérielle de ces modalités d'intervention. Les transformations discursives altèrent nécessairement le sens que l'on donne à des opérations inchangées puisqu'elles les insèrent dans des projets sociaux différents.

\section{Transformations des activités de contrôle social}

Peut-on déceler des transformations matérielles dans les activités de gestion? II ne s'agit pas ici de déterminer s'il y a moins d'État, ou plus d'État, mais d'identifier des tendances plus générales à partir de certaines transformations effectives qui semblent en voie de s'opérer.

On peut ramener à quelques questions simples les enjeux relatifs à l'exercice et aux transformations des formes de contrôle social : sur qui s'exercent les modalités particulières de contrôle? Comment ces cibles sont-elles identifiées? Qui assume la responsabilité du contrôle? Quelles formes spécifiques prend cette responsabilité? Bien que ces questions ne soient pas entièrement indépendantes les unes des autres, nous limiterons notre examen aux transformations liées de façon plus particulière à deux d'entre elles, la première portant sur les formes de responsabilité qui échoient aux diverses modalités de contröle et la seconde sur les modes d'alimentation du système pénal.

14. Mesures à caractère communautaire plutồt qu'institutionnel, stratégies de «diversion», mesures de substitution, par exemple. 
Bénévolat

La crise fiscale a donné lieu à certaines tentatives de rationalisation de l'intervention étatique dans le domaine du contrôle social. Les agences dont la fonction était principalement ou exclusivement d'agir auprès des administrés (d'effectuer le contrôle social, en quelque sorte) ont été, nous semble-t-il, les administrations les plus touchées par les mesures d'économie. Bien entendu, selon leur pouvoir relatif et leur capacité différente de mobilisation, ces agences $n$ 'ont pas toutes été affectées de la même façon par les coupures.

La rationalisation budgétaire a conduit l'État soit à cesser son intervention, soit à la déplacer, ou encore à en transférer la responsabilité matérielle. De cette manière, la réforme peut recevoir l'appui de différents groupes politiques. En obéissant au principe de la responsabilité individuelle lou à la responsabilité de petites unités sociales : famille, école, quartier, etc.l, elle satisfait les tenants du néo-libéralisme. Du même coup, l'importance qu'elle accorde à la communauté et aux groupes d'entraide de même que le transfert des responsabilités à des unités moins larges contentent les critiques de l'intervention étatique, qui lui reprochent d'être inappropriée, bureaucratique et inhumaine.

Ả cet égard, le déplacement des responsabilités reliées a certains types d'intervention directe lessentiellement la responsabilité de la prise en charge) a eu l'immense avantage de réconcilier idéologiquement des groupes divergents sur le plan politique.

Néanmoins, il faut dépasser le contenu discursif et examiner les limites de ces pratiques de déplacement et certains de leurs effets concrets. En ce qui a trait à l'implication de la communauté dans les interventions de contrôle social, il semble qu'elle atteint surtout des pratiques touchant les groupes sociaux les moins menaçants ou les plus faibles. Dans le domaine pénal, c'est au niveau de la gestion des sentences que s'opère le mouvement de déplacement. Quant au maintien de certaines activités ou à l'instauration de nouvelles formes d'intervention, cela ne se fait plus exclusivement par la création de nouvelles agences étatiques 
(État-intervenant). On observe plutôt un double mouvement qui s'inscrit directement dans la logique de la bienfaisance : obtention de services non rémunérés ou accroissement des services sans augmentation de salaire.

D'une part, l'accent est mis sur l'utilisation de plus en plus marquée du travail non rémunéré, qui se substitue au travail assumé par des agences étatiques ou qui accroît le volume de travail de ces mêmes agences. Par exemple, les nouvelles condamnations à des travaux communautaires impliquent l'accueil des personnes condamnées et la supervision de leurs travaux. L'encadrement, la supervision et le controle sont alors assumés, dans le cadre de contacts directs, par les membres des entreprises, institutions ou regroupements qui acceptent gratuitement de s'occuper des personnes condamnées. A ce titre, on peut donc supposer que les formes de contrôle n'ont pas disparu, comme certains ont pu le croire, ou que le contrôle s'est accru, comme d'autres le prétendent. C'est plutôt une substitution des gestionnaires du contrôle qui s'est produite.

$D^{\prime}$ autre part, le fonctionnement des groupes bénévoles ou sans but lucratif - dont le nombre va croissant dans la gestion pénale - est de plus en plus soumis à des contraintes relatives à leurs modes d'intervention (forme, fréquence) de même qu'au type et au nombre de leurs clients et clientes. La soumission à ces contraintes devient même une condition pour obtenir le financement nécessaire à la poursuite de leurs activités. Résultat : ces organisations bénévoles sont obligées d'assumer des tâches plus nombreuses ou plus difficiles dans un contexte plus réglementé et bureaucratique, tout en étant soumis aux mêmes normes de performance et sans que les ressources financières soient ajustées (parfois même elle sont réduites).

On constate donc que les transformations apportées à I'intervention étatique ne peuvent être décrites exclusivement comme des mesures de retrait ou d'accroissement. Elles se présentent aussi comme des formes spécifiques d'activités. Par exemple, on observe actuellement un accroissement de la supervision des activités d'intervention, le maintien ou la 
diminution de l'intervention directe et la transformation des agences subventionnées.

En résumé, le déplacement des responsabilités s'opère dans deux sens : vers les individus ou les familles qui prennent en charge la totalité ou une partie importante des interventions (soins, surveillance, etc.); vers des groupes dont le travail est totalement ou partiellement bénévole.

\section{Délation}

La nécessité de réduire, ou du moins de ne pas augmenter, certaines fractions de l'appareil étatique, couplée à l'idéologie de la responsabilité collective, a permis de développer ou de cautionner certaines formes de rapports entre les agences de contrôle social et les administrés.

L'application de tout règlement nécessite la mise en place des mécanismes de signalement des dérogations et de renvoi des cas vers les instances de supervision. Comme il est difficile de développer les agences de repérage, il faut instaurer d'autres moyens d'identifier les situations répressibles qui intéressent les agences. Par ailleurs, les tâches de surveillance accomplies bénévolement, surtout dans le domaine pénal, créent en elles-mêmes de nouvelles situations qui devraient être réprimées.

C'est ainsi que dans le domaine de la gestion des affaires pénales se développent ou se renforcent les mécanismes de signalement et de dénonciation. On peut ramener ces mécanismes à deux catégories : les interventions des citoyens ${ }^{15}$ et l'utilisation de délateurs techniques. Dans le premier cas, on se retrouve devant un éventail élargi de situations que les citoyens sont tenus légalement de dénoncer parce qu'elles relèvent en principe de la juridiction des agences pénales. II y a obligation de signaler et donc de déférer à des instances

15. Nous ne considérons pas spécifiquement ici le problème des délateurs, informateurs de police, agents provocateurs qui constituent, comme le dit Brodeur, 1988, «l'entreprise privée individuellew (p.186). Cette question est beaucoup trop complexe pour que nous l'abordions ici, et nous renvoyons les lecteurs aux ouvrages de cet auteur. 
judiciaires des situations ou des personnes que l'on présume devoir être réprimées. Si l'on fait exception de cas aussi évidents que l'obligation de prêter assistance à une personne en danger de mort, on reporte sur l'ensemble de la société l'épineux problème de déterminer ce qui constitue véritablement un objet de soupçon. Comment concilier cette tendance avec le respect élémentaire de la vie privée de chacun et chacune? Bien entendu, ces incitations à la dénonciation lesquelles ne sont nullement soumises aux règles de responsabilité personnelle et administrative (accountability) que l'on peut espérer de la part d'instances officielles - sont cautionnées par l'idéologie de la responsabilité individuelle.

Par ailleurs, les discours sur la rationalisation budgétaire, sur l'efficacité et sur la performance des systèmes entraînent une utilisation maximale des nouvelles techniques de surveillance et de dépistage. L'utilisation de bracelets électroniques pour la surveillance permanente des personnes condamnées ou sous observation judiciaire est une des propositions les plus disputées actuellement dans le domaine pénal ${ }^{10}$. L'usage, par les employeurs, de détecteurs de mensonges, de tests d'urine (dosage de drogue ou d'alcooll) s'inscrivent aussi dans cette optique de surveillance et de dénonciation. Enfin, la standardisation et la jonction des différentes banques de données alimentées par les agences étatiques (Justice, Santé, Assurance-chômage, Bien-être social, etc.) vont permettre non seulement d'identifier les personnes ayant des comportements légalement répréhensibles, mais pourront éventuellement donner lieu à la surveillance accrue de personnes ou de groupes qui, du point de vue des agences de surveillance, présentent supposément des facteurs de risque plus élevés. D'ailleurs, ce genre d'informations constitue la matière première d'agences de renseignements privées, très lucratives, et dont les services sont utilisés par les entreprises, les futurs employeurs ou quiconque souhaite en savoir davantage sur son voisin.

16. Pour un tour d'horizon de la situation à propos de cette question et un examen des problèmes posés par l'usage des bracelets électroniques, voir Landreville, 1987. 
Les pratiques de surveillance et de délation ne peuvent à long terme qu'altérer la nature des rapports interindividuels et des rapports sociaux. Cette altération est entretenue par le vide juridique dont ces pratiques sont l'objet. Ainsi, pour l'instant, on n'entrevoit pas les formes légalement acceptables qui pourraient encadrer de telles pratiques, pas plus qu'on ne prévoit les sanctions pour leur usage abusif ou malicieux.

\section{Conclusion}

L'évaluation des transformations que l'on observe dans les formes et modalités de contrôle social pose des problèmes complexes. La première difficulté est d'établir des distinctions entre transformations discursives et transformations matérielles. Si les changements discursifs au niveau des politiques ou des analyses relatives au système pénal méritent attention, elles ne prouvent pas pour autant que des changements concrets ont été apportés dans la pratique.

Bien qu'une telle distinction puisse être fondée pour tous les objets $d$ 'analyse sociologique ou politique, elle devient fondamentale dans le champ pénal en raison de l'incoherence bureaucratique et politique qui le caractérise. S'il y a parfois équivalence entre les transformations discursives et les changements concrets, il est fréquent que leur rapport en soit un de dissociation ou de dissonance. Dans certains cas, les premières $n$ 'indiquent que des orientations ou des possibilités sans déclencher de modifications effectives. Les seconds, même les plus visibles, ne sont pas toujours issus de discours explicites.

Les débats et les analyses sur la privatisation, qui ont occupé l'avant-scène depuis quelques années, constituent une excellente illustration du problème de dissonance. Ils permettent également de saisir la complexité des formes d'intervention étatique, même dans un domaine comme celui du pénal qui relève de la compétence exclusive de l'État. Ainsi, les tenants de la privatisation ont largement fait valoir les économies potentielles que le retrait des administrations publiques ferait faire aux contribuables. Or, le retrait de l'administration directe ne signifie pas pour autant la fin du 
financement par l'Etat. On substitue souvent une entreprise à l'État administrateur sans pour autant qu'il y ait cessation du transfert de fonds.

De plus, la privatisation de certaines activités pénales ne peut être ramenée à une simple question de finances. En effet, le transfert à des instances privées - plus ou moins organisées, avec ou sans but lucratif, bénévoles ou rémunérées - va provoquer le morcellement et la multiplication des agences de prise en charge. En conséquence, il nous semble que ces activités étatiques de coordination qui devront faire l'objet d'un financement croîtront au cours des prochaines années. Le contrôle des activités des groupes, organisations et agences permet $d$ 'assurer une standardisation des pratiques et le maintien d'une certaine cohérence dans l'orientation de ces pratiques ${ }^{17}$.

Le transfert de la responsabilité du maintien de l'ordre au secteur privé et, donc, le désinvestissement total de l'État en ce domaine seraient tout à fait impensables. Il s'agit là d'un élément fondamental de la reproduction des rapports sociaux. C'est donc sur le plan de la gestion directe que se font a

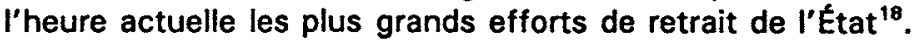
Ainsi, malgré un discours qui semble prôner la décroissance, on assiste paradoxalement à une expansion de l'appareil pénal qui s'opère par le biais de modalités parallèles. L'ajout de nouvelles unités de gestion ou d'intervention qui, même largement financées par l'état, sont techniquement indépendantes et qui se situent dans des normes et des cadres définis également par l'État permet de concilier les exigences liées à la rationalisation des finances publiques (couts généralement moins élevés, statut précaire) et la nécessité de maintenir le contrôle étatique.

17. C'est pourquoi certains groupes bénévoles préfèrent ne pas demander de soutien financier gouvernemental pour ne pas avoir à subir le carcan bureaucratique qui accompagne toujours l'octroi de subventions.

18. Comme le mentionne Belley, 1986, le supposé monopole étatique relatif à la pratique juridique est largement ebranlé par les pratiques alternatives ou paralleles qui se développent depuis maintenant plusieurs années (voir entre autres p. 30-31). 
Aussi retrouvons-nous de plus en plus aujourd'hui dans le paysage toutes sortes $d$ 'agences ou de formes $d$ 'intervention au statut hybride, si bien que les frontières entre le formel et l'informel, le privé et le public, l'étatique et le communautaire risquent de s'estomper. C'est de la définition que l'on donne au contenu de la catégorie étatique (par opposition à toute zone non étatique et non spécifiée) que dépend maintenant l'évaluation des transformations apportées aux pratiques de contrôle social : accroissement, diminution ou modification. A plus forte raison, cette définition détermine-t-elle l'appréciation que l'on veut faire des pratiques de contrôle qui impliquent le recours à la peine, lequel $n$ 'est plus au sens strict l'apanage exclusif de l'État. 
Bibliographie

BELLEY, J.-G., "L'État et la régulation juridique des sociétés globales. Pour une problématique du pluralisme juridiquen, Sociologie et sociétés, vol. XVIII, $n^{\circ} 1,1986$, p. 11-32.

BOTTOMS, A.E., "Some Neglected Features of Contemporary Penal Systemsw, dans GARLAND, D. et P. YOUNG, éd., The Power to Punish, London, Heinemann Educational Books, 1983, p. 166-202.

BRODEUR, J.-P., "Justice pénale et privatisation", dans BOISMENU, G. et J.-J. GLEIZAL, éd., Les mécanismes de régulation sociale. La justice, l'administration, la police, Montréal et Lyon, Boréal / Presses universitaires de Lyon, 1988, p. 183-231.

COHEN, S., "Social-Control Talk: Telling Stories about Correctional Change», dans GARLAND, D. et P. YOUNG, éd., The Power to Punish, London, Heinemann Educational Books, 1983, p. 101-129.

COHEN, S., "Taking Decentralization Seriously: Values, Visions and Policies", dans LOWMAN, J., MENZIES, R.J. et T.S. PALYS, ed., Transcarceration, Essays in the Sociology of Social Control, Aldershot, England, Gower, 1987, p. 358-379.

DORVIL, H., "La tolérance de la communauté à l'égard du malade mentaln, Santé mentale au Québec, vol. XII, $n^{\circ} 1$, 1987, p. 55-65.

GARLAND, D. et $P$. YOUNG, "Towards a Social Analysis of Penality", dans GARLAND, D. et P. YOUNG, ed., The Power to Punish, London, Heinemann Educational Books, 1983, p. 1-36.

GISLAIN, J.-J., "L'État et le marché : réflexions sur leur articulation institutionnelle», Interventions économiques, $n^{\circ} 17,1987$, p. 53-70.

GURVITCH, G., Eléments de sociologie juridique, Paris, AUBIER, 1940. 
KELK, C., "La surpopulation des prisons aux Pays-Bas», Déviance et Société, vol. XII, nْ 3, 1988, p. 303-309.

LABERGE, D., «Futur pénal, fiction pénale : débat autour des notions de privatisation et de désinvestissement étatiquen, Déviance et societé, vol. XII, $n^{\circ} 2,1988 a$, p. 169-175.

LABERGE, D., "D'une forme instituée à une autre : considérations sur l'analyse de la désinstitutionnalisation», Revue internationale d'action communautaire, $n^{\circ} 19 / 59,1988 b$, p. 33-41.

LANDREVILLE, P., «Surveiller et prévenir : I'assignation a domicile sous surveillance électroniquew, Déviance et société, vol. $X I, n^{\circ} 3,1987$, p. 253-272.

LANDREVILLE, P., «Quelques considérations à partir de la situation canadiennem, Déviance et Société, vol. XII, $n^{\circ}$ 3, 1988, p. 291-296.

LASCOUMES, P., «Du droit contrainte au droit ressource : la place du droit dans l'analyse des politiques publiquesn, Année sociologique, vol. 39, 1989a, p. 153-166.

LASCOUMES, P., «La pyramide éclatée : Boucles étranges et enchevêtrements dans la production et l'application du droit en France», Communication présentée au Colloque sur l'État-providence et la société civile, Ottawa, $1989 \mathrm{~b}$.

LEFEBVRE, Y., Psycauses, Montréal, Recherche suventionnée par le ministère de la Santé et du Bien-être social du Canada, 1985.

LEFEBVRE, Y., "Jalons pour une problématique québécoise de la désinstitutionnalisation», Santé mentale au Québec, vol. XII, n 1, 1987, p. 5-14.

LOWMAN, J., MENZIES, R.J.et T.S. PALYS, «Transcarceration and the Modern State of Penalityn, dans LOWMAN, J., MENZIES, R.J. et T.S. PALYS, ed., Transcarceration, Essays in the Sociology of Social Control, Aldershot, Angleterre, Gower, 1987, p. 1-15. 
MARX, G.T. et N. REICHMAN, «Routinizing the Discovery of Secrets : Computers as Informants", dans LOWMAN, J., MENZIES, R.J. et T.S. PALYS, éd., Transcarceration, Essays in the Sociology of Social Control, Aldershot, Angleterre, Gower, 1987, p. 188-208.

MATTHEWS, R., «Decarceration and Social Control : Fanta sies and Realities» dans LOWMAN, J., MENZIES, R.J. et T.S. PALYS, ed., Transcarceration, Essays in the Sociology of Social Control, Aldershot, Angleterre, Gower, 1987, p. 338-357.

PIRES, A.P. et P. LANDREVILLE, *Les recherches sur les sentences et le culte de la loin, l'Année sociologique, vol. 35, 1985, p. 83-113.

ROBERT, Ph., "De la criminologie du contrôle social à la sociologie pénale", l'Année sociologique, vol. 31, 1981, p. 253-283.

ROSS, E.A., Social Control : a Survey of the Foundations of Social Order, New York, Macmillan, 1901.

RUTHERFORD, A, . "La surpopulation pénitentiaire dans les prisons anglaises. Étude de cas d'une stratégie qui a échoué», Déviance et Société, vol. XII, $\mathrm{n}^{\circ} 3,1988$, p. 297-302.

VAILLANCOURT, $Y_{.,}$"La privatisation, une notion fourretout?" Déviance et société, vol. XII, $n^{\circ} 2,1988$, p. 177-182.

VAN DE KERCHOVE, M., "Les différentes formes de baisse de la pression juridique et leurs principaux enjeux", Cahiers de recherche sociologique, $n^{\circ} 13$, automne 1989, p. 11-30.

WARREN, C.A.B., "New Forms of Social Control. The Myth of Desinstitutionalization», American Behavioral Scientist, vol. 24, $n^{\circ} 6,1981$, p. 724-740.

YOUNG, P., "Sociology, the State and Penal Relations", dans GARLAND, D. et P. YOUNG, éd., The Power to Punish, Londres, Heinemann Educational Books, 1983, p. 84-100.

ZAMBROWSKY, J., "La privatisation ou le désinvestissement étatique dans la justice pénale, une politique pénale ou une fiction?" Déviance et société, vol. XII, $\mathrm{n}^{\circ} 2,1988$, p. 183-187. 